\title{
Deriving the loudness exponent from categorical judgments
}

\author{
KENNETH H. NORWICH and ELAD SAGI \\ University of Toronto, Toronto, Ontario, Canada
}

\begin{abstract}
The power function exponent for loudness is traditionally determined by means of a process of magnitude estimation. It is demonstrated in this paper that the exponent can also be obtained by using the procedure of absolute identification of sound intensity. It has been shown that subjects' responses to tones of a given intensity are distributed in a normal distribution whose variance depends on the range, $R$, over which the tones are distributed. By means of a standard statistical transformation, the normal density in log space is converted to the corresponding probability density in linear space. The power function exponent can then be obtained directly from the linear probability density. We also suggest that there is a direct relationship between the information calculated from experiments on absolute identification of sound intensity and the neurophysiological, poststimulus histogram measured in a nerve fiber in the auditory nerve.
\end{abstract}

A full logarithmic plot of magnitude estimation of loudness of tones against the physical intensity of these tones gives rise to a curve that is linear over much of its extent (S. S. Stevens, 1956). The slope of the linear portion of this curve is characteristic of the frequency of the tone studied and varies from above 0.4 for low and high frequencies to approximately 0.3 for tones at $1000 \mathrm{~Hz}$ (e.g., Marks, 1974; West, Ward, \& Khosla, 2000). We shall show here that the slope of this straight line (the power function exponent) can be obtained from the properties of a stimulus-response matrix constructed from an experiment on absolute identification of sound intensity.

Our stimulus-response matrix is a slight generalization of the one inaugurated by Garner and Hake (1951). It is designed to encode the results of an experiment on stimulus categorization, which includes absolute identification, and is described in detail by Norwich, Wong, and Sagi (1998). In brief summary, our experiments dealt with judgments of tone intensity and were conducted in the following manner: Tones of $1.5-\mathrm{sec}$ duration at $1000 \mathrm{~Hz}$ were delivered to subjects binaurally at intervals of $20 \mathrm{sec}$. The sound intensity was distributed uniformly from $1 \mathrm{~dB} H L$ to an upper bound of $R \mathrm{~dB} H L$. The subjects were trained to identify tones to the nearest decibel. Feedback was not required (Mori \& Ward, 1995). We confirmed the results of Mori and Ward repeatedly: Feedback to the subjects regarding the accuracy of their estimates did not improve overall performance. No more than 160 identifications were made by any subject in a

This work was supported by a grant from the Natural Sciences and Engineering Research Council of Canada. Correspondence should be addressed to K. H. Norwich, Institute of Biomaterials and Biomedical Engineering, University of Toronto, 4 Taddle Creek Road, Toronto, ON, M5S 3G9 Canada (e-mail: k.norwich@utoronto.ca). single day. Five hundred identifications were made by each subject for a given range, $R$. Each of 5 subjects was tested over several ranges $(1-10 \mathrm{~dB}, 1-30 \mathrm{~dB}, 1-50 \mathrm{~dB}$, 1-70 dB, and 1-90 dB).

Experiments over a given range could be analyzed by dividing the range into any reasonable number of categories of equal width. For example, an experiment carried out over the range 1-30 dB could be divided into 6 categories each of $5 \mathrm{~dB}$ in width, 10 categories $3 \mathrm{~dB}$ in width, 30 categories $1 \mathrm{~dB}$ in width, and so on. If there were $m$ stimulus categories and $m$ response categories, a stimulus-response matrix could be assembled in the usual way, with stimulus categories represented as rows and response categories as columns, as shown in Figure 1 . Then the entry in the $j$ th row and $k$ th column represents the number of times the subject identified a stimulus in the $j$ th category as belonging to the $k$ th category. Each of the $m$ stimulus categories has equal a priori probability.

Analysis of these experiments revealed that the distribution of responses to a tone of $\mu \mathrm{dB}$ was essentially normal with mean $\mu$ and variance $\sigma^{2}$. The mean, $\mu$, is known as the row mean, and the variance, $\sigma^{2}$, as the row variance. Row mean was measured in matrix units. Thus, if the range, $R$, were divided into $m$ categories, the mean responses for row $1,2, \ldots m$ would be approximately equal to $1,2, \ldots m$, respectively. Similarly, row variances are expressed in square matrix units. The row mean changed from row to row, but the row variance was found to be essentially constant throughout the entire matrix for a given subject over a given range. One can, therefore, calculate a mean row variance, which is characteristic of the entire matrix for the subject over that range.

In practice, one finds a distortion of the simple normal distribution of responses at the two ends of a row. This distortion has been called an anchor effect (Braida 


\begin{tabular}{|l|l|l|l|l|l|l|}
\hline & 1 & 2 & $\ldots$ & $k$ & $\ldots$ & $m$ \\
\hline 1 & $N_{11}$ & $N_{12}$ & & $N_{1 k}$ & & $N_{1 m}$ \\
\hline 2 & $N_{21}$ & $N_{22}$ & & $N_{2 k}$ & & $N_{2 m}$ \\
\hline$\vdots$ & & & & & & \\
\hline$j$ & $N_{j 1}$ & $N_{j 2}$ & & $N_{j k}$ & & $N_{j m}$ \\
\hline$\vdots$ & & & & & & \\
\hline$m$ & $N_{m 1}$ & $N_{m 2}$ & & $N_{m k}$ & & $N_{m m}$ \\
\hline
\end{tabular}

Figure 1. Stimulus-response matrix with $m$ stimulus and $m$ response categories. $N_{j k}$ is the number of times a stimulus in Category $j$ was identified by a response in Category $k$.

et al., 1984; Luce, Green, \& Weber, 1976). We devised a relatively simple means of removing the anchor effects (Sagi \& Norwich, 1999, in press), thus reinstating the normal distribution that would have been observed had the borders of the matrix not been present (see the Appendix). In the tables that follow, we represent the original mean row variance by $\sigma_{\text {eff }}^{2}$ and the row variance corrected for anchor distortion by $\sigma^{2}$. The two measures are similar but not exactly equal.

The Thurstonian hypothesis of constant row variance has permitted the development of a computer program that allows simulation of the performance of the subject for any range, $R$. The distribution of stimulus values was governed by the same uniform distribution used in the experiment. The responses were simulated by a Monte Carlo routine that generated normally distributed values about the mean response, using the Box-Müller algorithm. This simulator is an extension of the work of Houtsma (1983); the details of the simulator were reported by Wong and Norwich (1997). With the use of the simulator, it is possible to generate a stimulus-response matrix that is statistically indistinguishable from the original experimental matrix. Nearly identical values of the Shannon information can be calculated from either the original or the simulated matrix. Moreover, any number of trials can be generated by using the simulator, so one can obtain simulated matrices with $10^{4}$ or $10^{7}$ trials in a matter of a few minutes. The fastest version of the simulator is written in the $\mathrm{C}++$ computer language and runs within a Linux operating system.

It is well understood that when insufficient trials are conducted, estimates of the mutual or transmitted information from a stimulus-response matrix can be severely overestimated (Miller, 1955). The number of trials required for a statistically significant measurement of trans- mitted information is approximately five times the square of the number of categories in the stimulus-response matrix. A $20 \times 20$ matrix might require in excess of 2,000 trials for statistical significance. If one required estimation of intensity to the nearest decibel for a range of $90 \mathrm{~dB}, 40,000$ trials would be required. This poses no problems for $\mathrm{C}++/$ Linux, with which we can simulate, in a few minutes, sufficient trials for a $1,000 \times 1,000$ matrix and make all the required calculations.

Shannon's well-known equation for transmitted information, $I_{t}$, is

$$
I_{t}=H(Y)-H(Y \mid X),
$$

where $Y$ represents response data, and $X$, stimulus data. $H(Y)$ is the response entropy, and $H(Y \mid X)$ is the equivocation or error term. It was shown by Baird (1984) and Wong and Norwich (1997) that for large numbers of trials, $I_{t}$ is given by

$$
I_{t}=\ln m-\ln \sqrt{2 \pi e \sigma^{2}}
$$

which can be evaluated from the number of categories, $m$, and the corrected row variance, $\sigma^{2}$. We must note that Equation 2 does not take into account the anchor effect which, in principle, would increase the information slightly, but it suffices for heuristic purposes.

One can play an interesting and instructive game with either experimental or simulated data (since the two sets of data are indistinguishable). One enters the stimulusresponse pairs one by one into the stimulus-response matrix, and then calculates information after each trial entry. If we are simulating the data, we require as parameters only the values of $m$ and $\sigma^{2}$. Since we begin with only a single trial, then two, then three, and so on, we cannot use Equation 2 above, which is valid only for large numbers of trials. We must calculate information from the matrix using the methods of Garner and Hake (1951), which were adapted from Shannon's equations. We call these values calculated information to distinguish them from the transmitted information that is obtained from a large number of trials. When we plot calculated information against number of trials, $N$, we obtain a curve of characteristic shape, shown in Figure 2. The curve rises rapidly from zero to a peak that occurs when the number of trials just exceeds the value of $m$, and then declines slowly toward an asymptotic value, which is the transmitted information. This curve has been presented by the authors in previous publications.

\section{THE MATRIX OF MAXIMUM CARDINALITY}

The number of rows and columns, $m$, into which the stimulus-response matrix may be divided is, in principle, limited only by the number of experimental trials that are carried out. In a set-theoretical treatment of this problem (Sagi, Wong, \& Norwich, 2001), the matrix with the largest practicable value of $m$ has been termed the matrix of maximum cardinality, and the Shannon in- 


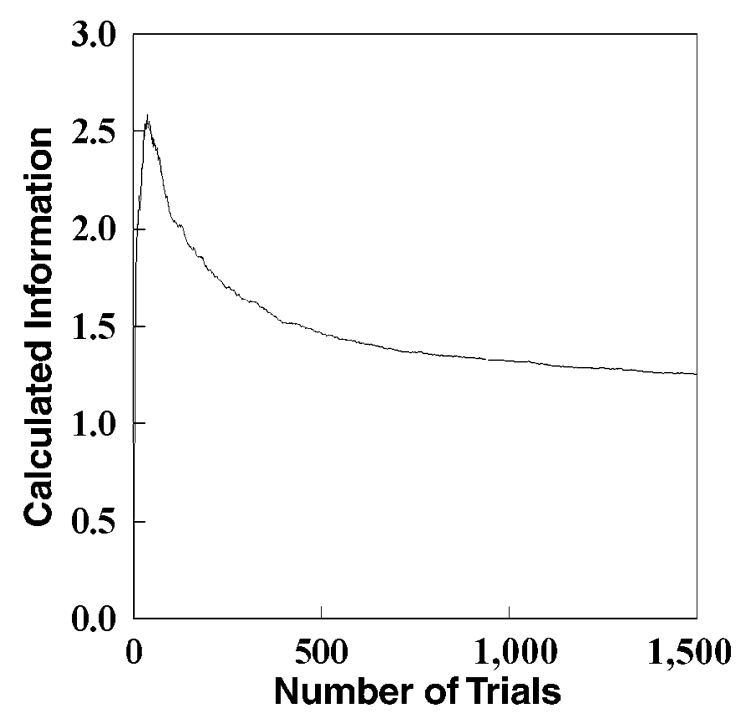

Figure 2. Calculated information (natural units) plotted against number of trials. Calculated information rises rapidly from zero to peak and then falls slowly toward its asymptotic value, which is the transmitted information. We could use measured or simulated data. This curve was simulated by using $\sigma^{2}=7.24$, with $m=$ 30 , for a maximum of 1,500 trials. These data will simulate an information curve whose peak is approximately equal to twice the asymptote.

formation obtained from such a matrix approaches a limiting value. In our experiments, the matrix of maximum cardinality is obtained by requiring the subject to estimate stimulus intensity to the nearest decibel. That is, $m=R \mathrm{~dB}$, where $R$ is the stimulus range fixed for a given experiment. A graph of calculated information against the number of trials is plotted from this matrix. We are particularly interested in the ratio of the peak to asymptotic information in this curve, because this ratio approaches a limiting value as $m$ becomes large. In this limit, this ratio depends on the sensory function of the human subject and the stimulus range used. By observation, the peak-to-asymptote ratios for curves of maximum cardinality approach a value that ranges from 1.8 to about 3.0 (Table 1).

\section{THE POWER LAW OF SENSATION EXPRESSED AS AN ENTROPY}

In the entropic view of sensation, magnitude of sensation is regarded as a measure of the entropy or uncertainty of the stimulus signal. Subjective magnitude can be expressed by means of the equation

$$
H=\frac{1}{2} \ln \left(1+\sigma_{s}^{2} / \sigma_{r}^{2}\right),
$$

where $\sigma_{s}^{2}$ is the variance of the stimulus signal as seen by the sensory receptor, and $\sigma_{r}^{2}$ is the variance of a reference signal that arises within the sensory receptor. For $\sigma_{s}^{2} / \sigma_{r}^{2} \ll 1,{ }^{1}$ we can write this equation in the form

$$
H=\frac{1}{2} \sigma_{s}^{2} / \sigma_{r}^{2}
$$

By hypothesis (Norwich, 1977, 1993),

$$
\sigma_{s}^{2} / \sigma_{r}^{2}=\gamma \mu_{s}^{n},
$$

where $\gamma$ is constant and $\mu_{s}$ is the stimulus mean in linear coordinates, so that

$$
H=\frac{1}{2} \gamma \mu_{s}^{n},
$$

which is an expression of the usual power law of sensation.

Equation 5 was introduced in 1977 by hypothesis: If this relationship between mean and variance prevailed, then the laws of sensation could be derived from the entropy of the stimulus. We shall show in the present work that this equation can be shown to be correct by using calculations from the stimulus-response matrix. Moreover, we shall be able to evaluate the ratio of variances $\sigma_{s}^{2} / \sigma_{r}^{2}$ from the matrix and hence evaluate the exponent, $n$, which is the power function exponent for hearing.

\section{POWER FUNCTION EXPONENTS FROM THE STIMULUS-RESPONSE MATRIX}

The power function exponent for audition, $n$, is calculated psychophysically by measuring the slope of the linear portion of the curve obtained when the logarithm of subjective magnitude is plotted against the logarithm of stimulus intensity. It will now be shown that this exponent can be estimated from the stimulus-response matrix, by expressing $\sigma_{s}^{2}$ and $\sigma_{r}^{2}$ (which define the psy-

\begin{tabular}{|c|c|c|c|c|c|c|}
\hline Range (dB) & $N_{\text {peak }}$ & $I_{\text {peak }}$ & $\sigma_{\mathrm{eff}}^{2}\left(\mathrm{~dB}^{2}\right)$ & $\sigma^{2}\left(\mathrm{~dB}^{2}\right)$ & $I_{t}$ & $I_{\text {peak }} / I_{t}$ \\
\hline \multicolumn{7}{|c|}{ Subject W[25] } \\
\hline $1-10$ & 10 & 1.557 & 2.207 & 3.063 & 0.565 & 2.76 \\
\hline $1-30$ & 24 & 2.477 & 6.270 & 7.324 & 1.107 & 2.24 \\
\hline $1-50$ & 53 & 2.963 & 13.874 & 15.897 & 1.215 & 2.44 \\
\hline $1-70$ & 60 & 3.114 & 19.437 & 21.749 & 1.376 & 2.26 \\
\hline $1-90$ & 90 & 3.375 & 31.158 & 34.798 & 1.391 & 2.43 \\
\hline \multicolumn{7}{|c|}{ Subject J[18] } \\
\hline $1-10$ & 11 & 1.594 & 2.061 & 2.814 & 0.597 & 2.67 \\
\hline $1-30$ & 24 & 2.694 & 8.205 & 9.849 & 0.979 & 2.75 \\
\hline $1-50$ & 50 & 3.111 & 19.656 & 23.229 & 1.050 & 2.96 \\
\hline $1-70$ & 76 & 3.371 & 26.623 & 30.454 & 1.226 & 2.75 \\
\hline $1-90$ & 116 & 3.601 & 25.831 & 28.533 & 1.481 & 2.43 \\
\hline \multicolumn{7}{|c|}{ Subject E[22] } \\
\hline $1-10$ & 9 & 1.581 & 1.904 & 2.556 & 0.634 & 2.49 \\
\hline $1-30$ & 43 & 2.499 & 6.029 & 7.018 & 1.125 & 2.22 \\
\hline $1-50$ & 25 & 2.886 & 13.546 & 15.492 & 1.227 & 2.35 \\
\hline $1-90$ & 154 & 3.332 & 28.623 & 31.803 & 1.432 & 2.33 \\
\hline \multicolumn{7}{|c|}{ Subject C[19] } \\
\hline $1-10$ & 33 & 1.573 & 1.218 & 1.516 & 0.843 & 1.87 \\
\hline $1-30$ & 40 & 2.454 & 6.542 & 7.672 & 1.087 & 2.26 \\
\hline $1-90$ & 71 & 3.418 & 41.680 & 47.481 & 1.251 & 2.78 \\
\hline \multicolumn{7}{|c|}{ Subject R[19] } \\
\hline $1-10$ & 13 & 1.458 & 1.348 & 1.702 & 0.795 & 1.83 \\
\hline $1-30$ & 41 & 2.483 & 5.067 & 5.812 & 1.208 & 2.06 \\
\hline $1-90$ & 108 & 3.467 & 24.884 & 27.431 & 1.499 & 2.31 \\
\hline
\end{tabular}

Table 1

Results from Identification Experiments Using 1000-Hz Tones for Each Subject

Note-Information is given in natural units. 
chophysical function) as functions of $\sigma^{2}$ and $\mu$ (which are measurable properties of the matrix). There are two components in the calculation of the exponent. The first involves a statistical transformation of variables; the second requires only a least squares regression line.

\section{Experiment}

Identification experiments were conducted with $1000-\mathrm{Hz}$ tones for 5 subjects between the ages of $18-25$ years. For each subject, experiments were conducted for three to five stimulus ranges measured in units of $\mathrm{dB} \mathrm{HL}$ (i.e., decibels above a population threshold). For each range, 500 experimental trials were conducted and compiled into a stimulus-response matrix (Figure 1). The subjects were required to identify stimulus intensity to the nearest decibel for any given range, $R$, so that the stimulus-response matrix would be an $R \times R$ matrix, or the matrix of maximum cardinality. The Shannon information was calculated for each matrix as a function of the number of trials and produced a curve of characteristic shape (Figure 2). These curves peaked after $N_{\text {peak }}$ trials at a value of $I_{\text {peak }}$ natural units (n.u.) and subsequently approached an asymptotic value of $I_{t}$ n.u., which is the transmitted information. For each subject, the ratio of peak information to transmitted information, $I_{\text {peak }} / I_{t}$, is roughly $2: 1$. From the matrix of maximum cardinality, the arithmetic mean of the row variances, $\sigma_{\text {eff }}^{2}$, was measured. The arithmetic mean row variance was then used to determine the variance, $\sigma^{2}$, that underlies a subject's response, free from anchor effects (see the Appendix). We must stress that $\sigma_{\text {eff }}^{2}$, and subsequently $\sigma^{2}$, were obtained completely from experimental data (i.e., without the use of simulation). These results are reported for each subject in Table 1. The manner in which the exponent was extracted for each subject with the use of only the obtained values of $\sigma^{2}\left(\mathrm{~dB}^{2}\right)$ and $R(\mathrm{~dB})$ will be explained below.

\section{Statistical Component}

The requirement for statistical transformation of data arises from the difference in the "spaces" in which the two cardinal variances are measured. The variance, $\sigma^{2}$, which is the corrected row variance of the stimulus-response matrix, is measured in log space. That is, within an experiment on judgment of intensities, sound intensities are measured in decibels, which are a logarithmic measure. The standard deviation at each row, $\sigma$, is therefore expressed in decibels. However, $\sigma_{s}^{2}$, as expressed in Equations 4 and 5, is a variance in ordinary linear space. It represents the variance in the signal intensity as experienced by the sensory receptors - in this case, the inner hair cells on the basilar membrane within the cochlea. Both of these variances refer to the same physical signal in that they both encode the uncertainty of the perceiving system with regard to its physical stimulus. However, they have been expressed in different coordinates. Part of our task will be to relate the two variances mathematically.
The distribution of responses within each row of the stimulus-response matrix is discrete in nature. However, as discussed above, it may be well approximated by the continuous normal distribution within log or decibel space. The appropriate statistical test for normality of a given row distribution was given in Figure 5 of Norwich et al. (1998). If we represent the row mean by $\mu(\mathrm{dB})$, the variance by $\sigma^{2}\left(\mathrm{~dB}^{2}\right)$, and the statistical variable giving the probability density along a row by $x(\mathrm{~dB})$, then the normal distribution is expressed by the familiar equation

$$
\operatorname{Normal}(x)=\frac{1}{\sqrt{2 \pi \sigma^{2}}} e^{-(x-\mu)^{2} / 2 \sigma^{2}} .
$$

The value of the mean of the normal density for a given row is referred to as the stimulus intensity for that row. For example, when one works with the stimulus range 1-90 dB using 90 categories, matrix row 30 is characterized by $\mu=30 \mathrm{~dB}$. However, for a given range, $R$, the mean stimulus intensity presented to the listener is

$$
\mu=\frac{R}{2} \text {. }
$$

In order to obtain an expression for the linear parameter, $\sigma_{s}^{2}$, in terms of the logarithmic parameter, $\sigma^{2}$, we convert variables to the linear $y$ from the logarithmic $x$. That is,

$$
x=10 \log _{10} y .
$$

By employing the transformation theorem of statistics, we obtain

$$
\text { Linear }(y)=\frac{\alpha}{y \sqrt{2 \pi \sigma^{2}}} e^{-(\alpha \ln y-\mu)^{2} / 2 \sigma^{2}},
$$

where Linear $(y)$ is the probability density function in linear space corresponding to the normal distribution in decibel space, and

$$
\alpha=\frac{10}{\ln 10} .
$$

The remainder of the calculations is quite routine. If we represent the mean intensity in linear space by $\mu_{s}$ (corresponding to $\mu$ in decibel space), and recall that $\sigma_{s}$ in linear space corresponds to $\sigma$ in decibel space, then

$$
\mu_{s}=\exp \left(\frac{2 \mu \alpha+\sigma^{2}}{2 \alpha^{2}}\right)
$$

and

$$
\sigma_{s}^{2}=\mu_{s}^{2}\left[\exp \left(\frac{\sigma^{2}}{\alpha^{2}}\right)-1\right]
$$

or

$$
\left(\frac{\sigma_{s}}{\mu_{s}}\right)^{2}=\left[\exp \left(\frac{\sigma^{2}}{\alpha^{2}}\right)-1\right],
$$

which we shall use in the next section. The quantity $\sigma_{s} / \mu_{s}$ is commonly referred to in statistics as the coefficient of variation. The latter three equations express the mean 
Table 2

Loudness Function Exponents Derived From Identification Experiments for Tones at $1000 \mathrm{~Hz}$

\begin{tabular}{lccccr}
\hline & \multicolumn{5}{c}{ Subject } \\
\cline { 2 - 6 } & $\mathrm{W}$ & $\mathrm{J}$ & $\mathrm{E}$ & $\mathrm{C}$ & $\mathrm{R}$ \\
\hline Exponent $(n)$ & 0.332 & 0.322 & 0.336 & 0.452 & 0.344 \\
\hline
\end{tabular}

and variance of the linear probability density in terms of the mean and variance of the logarithmic density.

Equation 12 can be expressed in a simpler form. Taking logarithms of both sides,

$$
\ln \mu_{s}=\frac{\mu}{\alpha}+\frac{\sigma^{2}}{2 \alpha^{2}} .
$$

This equation is largely intuitive. If we regard only the first term on the right-hand side, then $\mu_{s} \approx e^{\mu / \alpha}=10^{\mu / 10}=$ $10^{R / 20}$. For example, when one deals with a matrix row whose mean in decibels is equal to 15 , the value of the mean of the corresponding linear distribution is approximately equal to $10^{15 / 10}=10^{1.5}$. In most of the work that follows, however, we use the full expression given in Equation 15.

\section{Power Function Exponent by Least Squares Regression}

To produce consonance with the entropy theory, we shall take the mean reference intensity in linear space to be equal to the mean signal intensity in linear space. That is,

$$
\mu_{r}=\mu_{s} .
$$

By conjecture, we shall take the decibel variance corresponding to the reference tone to be constant. Then, according to Equation 13, we have

$$
\sigma_{r}^{2}=\mu_{s}^{2} / K
$$

This equation represents a slight refinement of the procedure used to derive the entropy equation in Norwich (1977) (where $\sigma_{r}^{2}$ was assumed to be independent of $\mu_{s}$ ), but leads to the same final equation. Therefore, the ratio of linear variances, $\sigma_{s}^{2} / \sigma_{r}^{2}$, that appears in the entropy Equation 4 , is given by

$$
\frac{\sigma_{s}^{2}}{\sigma_{r}^{2}}=K\left[\exp \left(\frac{\sigma^{2}}{\alpha^{2}}\right)-1\right]=K\left(\frac{\sigma_{s}}{\mu_{s}}\right)^{2} .
$$

In other words, the ratio of linear variances that appears in the entropy equation is proportional to the square of the coefficient of variation derived statistically from the matrix.

What remains is an empirical exercise to show that for the stimulus-response matrix of greatest cardinality, the ratio $\sigma_{s}^{2} / \sigma_{r}^{2}$ is a power function depending solely on the mean stimulus intensity, $\mu_{s}$. For each of 5 subjects, we obtained the values of $\sigma^{2}$ corresponding to the values of $\mu$ at each range (Equation 8, Table 1). Subsequently, values of $\mu_{s}$ and $\sigma_{s}^{2}$ can be calculated for each range using Equation 12 and Equation 13, respectively. Hence, the values of $\left(\sigma_{s} / \mu_{s}\right)^{2}$ can be calculated and plotted against $\mu_{s}$. A good straight line can be fitted for $\ln \left(\sigma_{s} / \mu_{s}\right)^{2}=$ $\ln \left[\exp \left(\sigma^{2} / \alpha^{2}\right)-1\right]$ plotted against $\ln \mu_{s}$, as shown in Figure 3 , for each subject.

By way of example, the equation of this straight line for Subject $\mathrm{W}$ is given by

$$
\left[\exp \left(\frac{\sigma^{2}}{\alpha^{2}}\right)-1\right]=0.134 \mu_{s}^{0.332}
$$

or, from Equation 18, by

$$
\sigma_{s}^{2} / \sigma_{r}^{2} \propto \mu_{s}^{n}=0.134 K \mu_{s}^{0.332} .
$$

Introducing this value for $\sigma_{s}^{2} / \sigma_{r}^{2}$ into Equation 3 from the entropy theory we obtain

$$
H=\frac{1}{2} \ln \left(1+0.134 K \mu_{s}^{0.332}\right) .
$$

$H$ has been taken here as a measure of neural firing rate, which can, over a limited range, describe the loudness, $L$, of a tone. That is,

$$
L=\frac{1}{2} k \ln \left(1+0.134 K \mu_{s}^{0.332}\right) .
$$

When $0.134 K \mu_{s}^{0.332}$ remains less than unity, the first order term in the Taylor series expansion of the logarithm gives

$$
L=\left(\frac{1}{2} 0.134 K k\right) \mu_{s}^{0.332} .
$$

That is, for Subject W, the loudness function measured from the stimulus-response matrix of greatest cardinality is a power function with an exponent equal to 0.33 for a $1000-\mathrm{Hz}$ tone.

Similar results are found with data from the other subjects and are presented in Table 2 and Figure 3. In accordance with the results of West et al. (2000), we found that the loudness exponent is not necessarily fixed and can vary across subjects. To further determine whether the loudness exponent derived from categorical judgments is, in fact, equivalent to the exponent derived from the magnitude estimation data, one would have to show that the exponent differs significantly at different stimulus frequencies for a given subject. Current studies in our laboratory are focussed on the changes in the matrix-derived exponent at different auditory frequencies.

\section{DISCUSSION}

We have pursued the exponent of the loudness function somewhat monolithically in this work, giving scant attention to other important variables that affect the loudness function. J. C. Stevens and Marks (1999) have directed attention to the triad of prime factors, exponents, intercepts, and thresholds, all of which must be considered for a comprehensive treatment of the power law of sensation. West et al. (2000) have explored the phenomenon of learned scaling, in which subjects can 

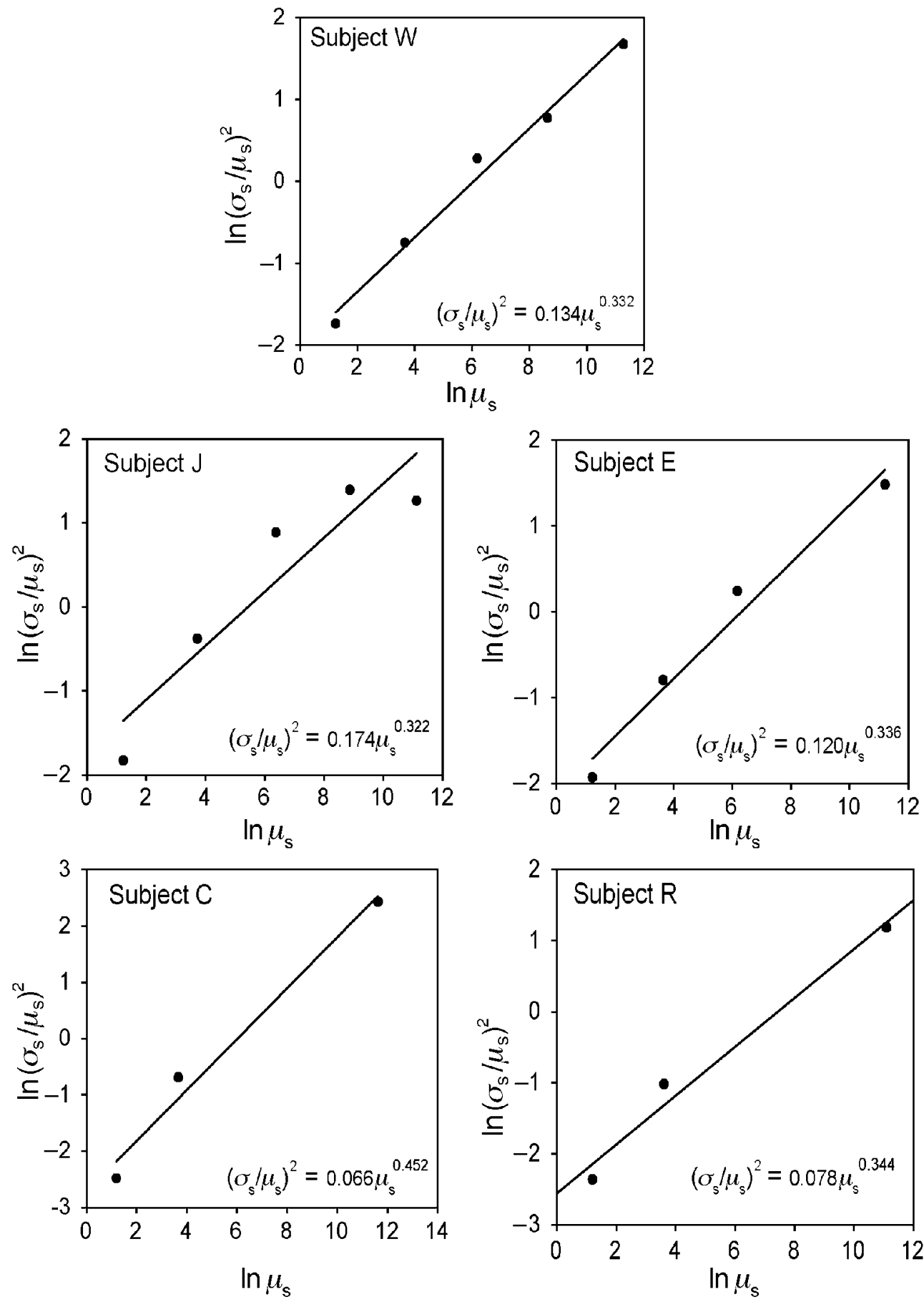

Figure 3. Relationship between linear mean, $\mu_{s}$, and linear variance, $\sigma_{s}^{2}$, for auditory intensity for Subjects $\mathbf{W}, \mathbf{J}, \mathbf{E}, \mathbf{C}$, and $\mathbf{R}$. The square of the coefficient of variation, (i.e., $\left.\sigma_{s} / \mu_{s}\right)$ is plotted against $\mu_{s}$ in a full$\log$ plot. The slope of the regression line gives the value of the power function exponent for a $1000-\mathrm{Hz}$ tone as expressed in Equation 20.

produce not just one, but a spectrum of power function exponents. Their work, when viewed in the context of our present study, opens up a number of interesting possibilities. The subjects, with appropriate instruction, could, in principle, alter the exponent calculated from their stimulus-response matrices. Subjects might, voluntarily or involuntarily, increase their errors in identification, hence increasing the measured value of $\sigma^{2}$, thereby 
decreasing the transmitted information. They cannot, however, decrease their errors below those reported here, since their performance was already optimal. It can be shown, though, that by the artifice of increasing errors in judgment, subjects can selectively either increase or decrease the calculated value of the exponent, $n$, in parallel with the results of West et al.

This flexibility in the measurement of $n$ poses some difficulties, since one could very well argue that the exponent is completely contrived. If, however, one agrees that the exponent can be obtained naturally, for example under unconstrained conditions as demonstrated by S. S. Stevens (1956), we can understand the effects of experimental constraints as altering this "natural" underlying exponent. By assuming that such a value does exist, one can then argue that this value should be obtainable from differing psychophysical methods that measure the same underlying sensory structure (Baird, 1970). Hence, we consider our approach of deriving the loudness exponent from categorical judgment as an instance of a more general principle.

When the law of sensation is expressed in its entropic form in Equation 4, it possesses a great deal of explanatory and predictive value (Norwich, 1993, pp. 248-249). However, in order to identify Equation 4 with the standard form of the power law (i.e., Equation 6), we must either assume the validity of Equation 5 or demonstrate its validity empirically. In this paper, we have attempted to do the latter. Is it, in fact, true that $\sigma_{s}^{2} / \sigma_{r}^{2} \propto \mu_{s}^{n}$ ? This question was posed by Norwich (1977), and we seem now to be able to answer with some assurance because, with the aid of the stimulus-response matrix, we are able for the first time to measure both quantities, $\sigma_{s}^{2} / \sigma_{r}^{2}$ and $\mu_{s}^{n}$.

In order to demonstrate the truth of Equation 5, we first identified the variance, $\sigma_{s}^{2}$, embodying fluctuation of the stimulus signal at the sensory receptor, with $\sigma^{2}$, the variance defining the error in a subject's identification of an unknown stimulus. This basic assumption implies that errors in identification of stimuli begin peripherally, at the level of the sensory receptor, rather than centrally-for example, at the cerebral cortex. In contrast, other investigators have held that errors in identification of stimuli are incurred mainly centrally owing to factors such as memory and/or attention. In particular, these factors are thought to relate to the increase of $\sigma^{2}$ with an increasing stimulus range (Braida \& Durlach, 1972). The latter approach, however, arises from an interpretation of modeling constraints as opposed to a derivation from first principles (Luce et al., 1976). In our model, we demonstrate an inherent relationship between $\sigma^{2}$ and $\sigma_{s}^{2}$. Furthermore, our validation of Equation 5 allows us to extract the loudness exponent from the increase of $\sigma^{2}$ with stimulus range.

The variance $\sigma_{s}^{2}$ is produced in linear space, whereas $\sigma^{2}$ is measured in decibel space. By making the necessary statistical transformations, we were able to relate these variances through Equation 13. Similarly, the ratio of linear variances, $\sigma_{s}^{2} / \sigma_{r}^{2}$, was related to decibel variance, $\sigma^{2}$, through Equation 18. The only step that re- mained in order to demonstrate the truth of Equation 5 was to show that the middle member of Equation 18, $\exp \left(\sigma^{2} / \alpha^{2}\right)-1$, was proportional to $\mu^{n}$, where $n$ is some positive exponent. The latter step is confined totally to calculations made on the stimulus-response matrix obtained from experiment.

Although $\sigma^{2}$, the anchor-free row variance of the stimulus-response matrix, if measured in units of number of categories, will change as $m$ changes, $\sigma^{2}$ will remain constant for a given range, $R$, irrespective of $m$, if measured in units of decibels. Similarly, $\mu_{s}$, as measured by Equation 12, will remain constant for a given range, independent of the number of categories. So we are free to utilize any categorical division for purposes of estimating the power function exponent. However, the greater the value of $m$ utilized, the greater the resolution in determination of $\sigma^{2}$. Therefore, we recommend using the matrix of maximum cardinality permitted by the measuring device (audiometer in this case). Furthermore, measurement of $\sigma^{2}$ from experimental data can be achieved with a far fewer number of trials than would be required for a significant estimation of the transmitted information. For example, our measure of $\sigma^{2}$, for each subject at a stimulus range of $1-90 \mathrm{~dB}$, was obtained by using only 500 experimental trials, without the need for simulation.

It is noted that the loudness function generated by the stimulus-response matrix does not demonstrate the characteristic downward deflection for low stimulus values observed in measurements of magnitude estimation. This downward deflection could be demonstrated by matrix techniques only if a substantial number of experiments were conducted in the lower stimulus ranges (e.g., 1-5 dB, 1-7 dB, etc.). We did not scrutinize stimuli in this range. As is demonstrated below, however, the required downward deflection can be simulated.

Within the entropic view of sensation, the variable time since onset of stimulus is replaced by the more fundamental variable number of trials made. It may be that the two variables differ only by a scaling factor. In experiments on categorical judgment, trials (sampling of the stimulus continuum) are made slowly, which is an artifice of experimental design. In the ear itself, trials or sampling proceeds much more rapidly. The results, however, are the same: the extraction of loudness information from the stimulus signal.

In experiments on categorical judgments, we continue to regard each range of stimuli as a single intensity. That is, we take a range of stimuli spanning, for example, 1-30 dB, as representative of a single stimulus, distributed normally about a mean of $15 \mathrm{~dB}$. In this way, the experiment on categorical judgment simulates a pure tone with an intensity that varies in accordance with statistical principles above and below a $15-\mathrm{dB}$ mean of the corresponding linear distribution (Equation 12), or about $10^{1.5}$ intensity unit.

We are now in a position to discuss how the extraction of the loudness function exponent from the stimulusresponse matrix permits the mapping of the curve of cal- 
culated information onto the neurophysiology curve of adaptation. By comparing the information curve in Figure 2 with the poststimulus time (PST) histograms in Figures 4A-4C, we can observe the similarity. PST histograms at different stimulus intensities correspond to information curves made for different ranges of stimuli (i.e., different means). The ratio of the peak amplitude of each curve to its asymptotic amplitude varies from about 1.5 to about 2.5 in both types of curve (Table 1 and Figures $4 \mathrm{~A}-4 \mathrm{C})$. This constraint in the peak to asymptote ratio to about the value of $2: 1$ is a property of the biological information function. That is, freely generated information curves can have ratios that are indefinitely greater or smaller. The constraint to $2: 1$ is produced by the auditory system itself.

One should note that the $2: 1$ constraint is independent of stimulus range in absolute identification and independent of stimulus intensity in neurophysiological adaptation. We shall now see that the loudness function exponent arises from this $2: 1$ constraint.

Figure 5A contains a simulated graph of information plotted against number of trials. It has been plotted by using the statistical properties of the information curve (Carlton, 1969). The parameters of the curve have been selected so that the peak will be twice the amplitude of the asymptote ( $2: 1$ curve). This curve is a generic curve
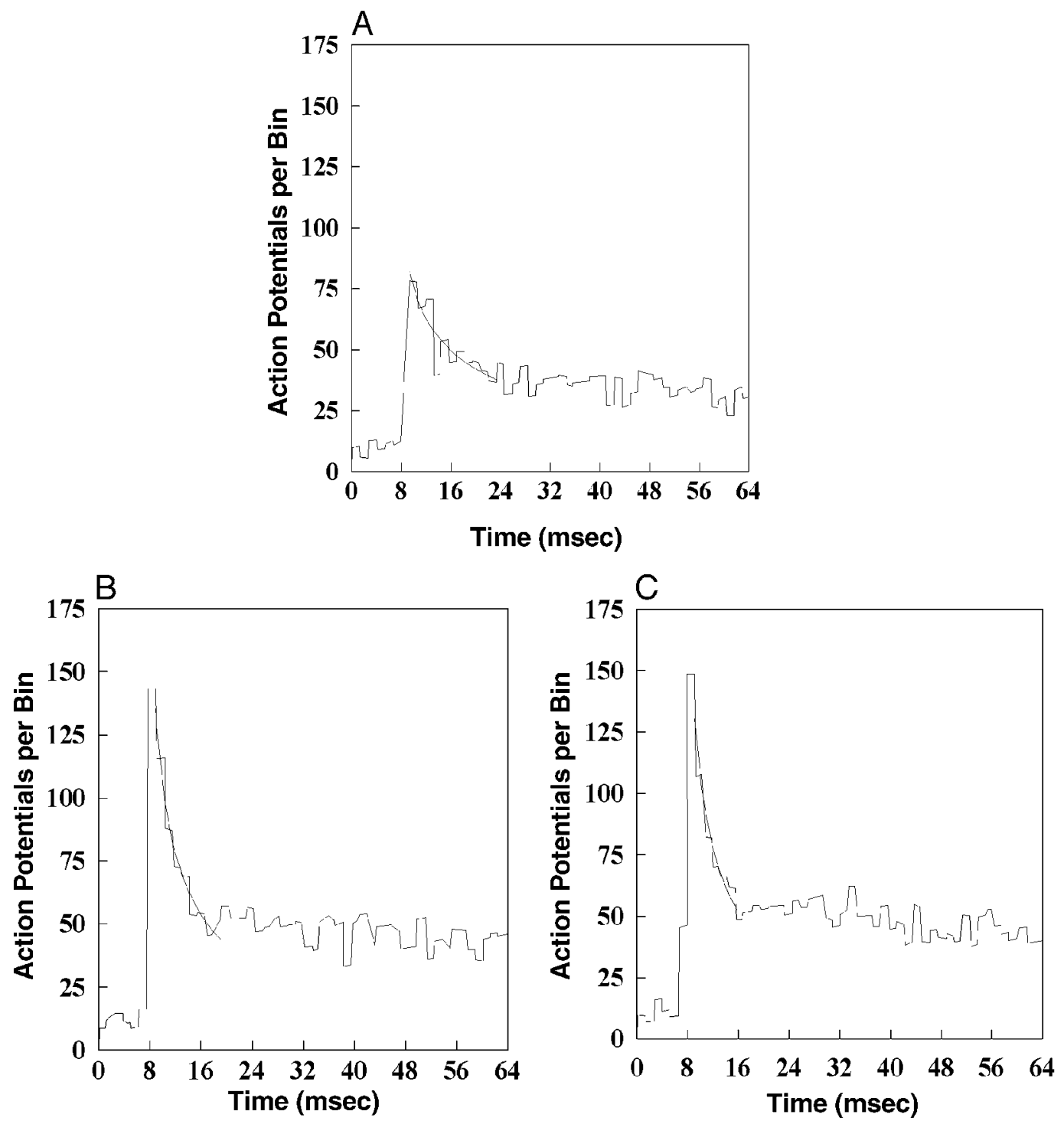

Figure 4. Poststimulus-time histograms from single auditory nerve fibers in anesthetized Mongolian gerbils. The histograms have been reproduced approximately from those of Smith and Brachman (1980). Panels A, B, and $\mathrm{C}$ are the responses to auditory stimuli of 11, 21, and $53 \mathrm{~dB}$, respectively. The smooth curves drawn through the initial descending portion of the histograms have been calculated from the entropy Equation 4 . The time dependence is taken from Equation 24 by using the central limit theorem (Norwich, 1993, Equation 11.4). 

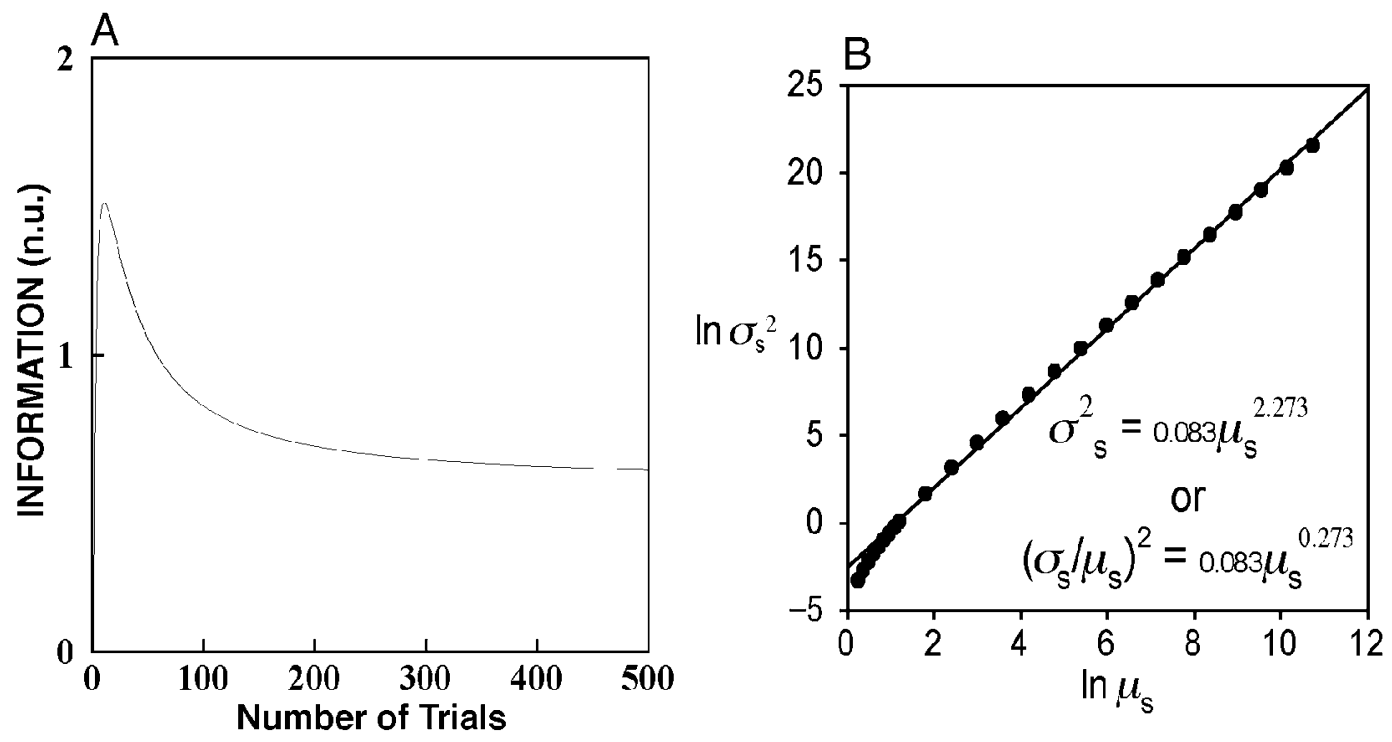

Figure 5. (A) Calculated information plotted against number of trials by using the expectation of the calculated information. Unique values of $R$ and $\sigma^{2}$ were selected so that the peak value of calculated information was twice the amplitude of the asymptotic value (i.e., 2:1). (B) Full-log plot of linear variance, $\sigma_{s}^{2}$, against the linear mean, $\mu_{s}$ (Equations 13 and 15), using only values of $R$ and $\sigma^{2}$ that uphold the 2:1 criterion. The slope of the linear portion of the curve as presented is 2.273. By dividing through by the square of the linear mean as described in Equation 18, the loudness function exponent is measured at 0.273 .

from which all experimental information curves may be derived, and from which all neurophysiological PST histograms may be simulated. By specifying a value for range, $R$, only a single value for variance $\sigma^{2}$ is permitted if the curve is to retain its $2: 1$ property. As one increases the stimulus range, one must also increase $\sigma^{2}$. Hence, the $2: 1$ criterion can be used to obtain increasing values of $\sigma^{2}$ as the stimulus range is increased.

Furthermore, by using the techniques outlined in this paper, one can then extract the loudness function exponent from these values. This is demonstrated in Figure $5 \mathrm{~B}$, where the linear variance, $\sigma_{s}^{2}$, is plotted against linear mean, $\mu_{s}$, in a full-log plot using values of $\sigma^{2}$ that conform to the $2: 1$ criterion. In this figure, the linear portion of the increase is evaluated at 2.273. To obtain the loudness function exponent, one must divide by the square of the linear mean as expressed in Equation 14, which leaves one with an exponent value of 0.273 . One should also note the downward deflection for low stimulus values, characteristic of the loudness function.

Following a slightly more advanced treatment of the problem of matching or identifying the properties of stimulus-response matrices with the entropic form of the psychophysical law, we are led, paradoxically, to a somewhat simpler method for computing the power function exponent. By combining Equations 3 and 5, and multiplying by a scaling constant, $k$, we obtain

$$
L=k H=\frac{1}{2} k \ln \left(1+\gamma \mu_{s}^{n}\right),
$$

which relates loudness, $L$, to stimulus magnitude, $\mu_{s}$ (Norwich, 1977, 1993).
Leaving entropy theory and returning to the stimulusresponse matrix, we invert Equation 13, to obtain

$$
\sigma^{2}=\alpha^{2} \ln \left(1+\sigma_{s}^{2} / \mu_{s}^{2}\right) \text {. }
$$

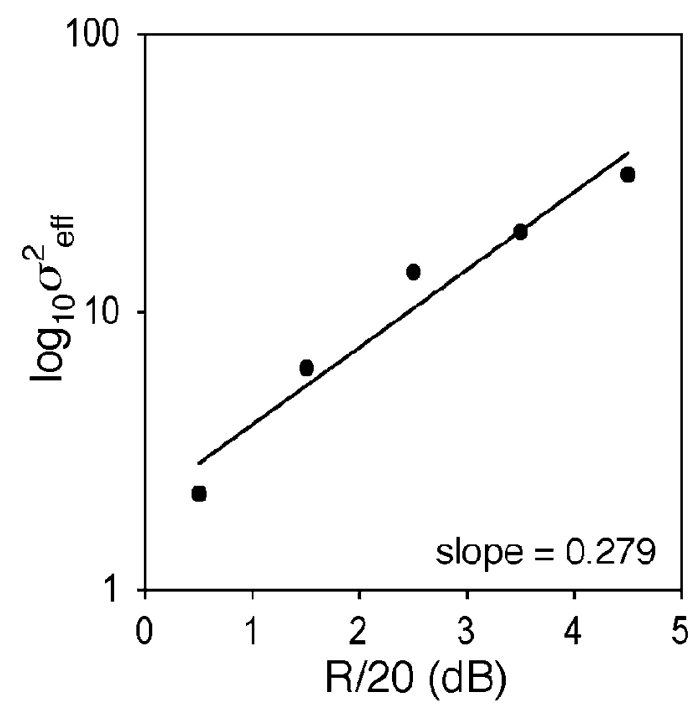

Figure 6. Rapid and simple estimate of the power function. The $\log$ of $\sigma_{\text {eff }}^{2}$ is plotted against the log of estimated $\mu_{s}$ for Subject W. The slope of the regression line equals 0.28 , which can be taken as an estimate of the value of the power function exponent, $n$. The quantity $\sigma_{\text {eff }}^{2}$ is the measured mean row variance of the stimulusresponse matrix, uncorrected for anchor effects; and $\mu_{s}$ is the value of the mean for each matrix calculated simply as $10^{R / 20}$, where $R$ is the range for the matrix. 
By introducing Equation 17, we can write the latter equation in the form

$$
\sigma^{2}=\alpha^{2} \ln \left(1+\sigma_{s}^{2} / K \sigma_{r}^{2}\right)
$$

or, by employing Equation 20,

$$
\sigma^{2}=\alpha^{2} \ln \left(1+\gamma^{\prime} \mu_{s}^{n}\right)
$$

where $\gamma^{\prime}$ is constant. Equation 24 is derived completely from entropy theory, whereas Equation 26 is obtained completely from an information-theoretic analysis of the stimulus-response matrix. However, we can now identify the mean row variance, $\sigma^{2}$, with loudness, $L$, which is, in itself, rather unexpected. This identification of $\sigma^{2}$ with loudness emerges here as a purely mathematical consequence of comparing two equations. However, the concept that loudness is a function of uncertainty, or error, is fundamental to the entropy view of sensation. In fact, the symmetry between Equation 24 and Equation 26 implies that the overall perceived loudness becomes encoded in the errors of a subject's response in a given identification experiment.

Equation 24 permits us to estimate the power function exponent quite simply by using only elementary operations on the stimulus-response matrix. By using the usual Taylor series expansion for smaller values of $\mu_{s}$, we can see that loudness, now encoded by $\sigma^{2}$, will be expressed as a simple power function of $\mu_{s}$, the mean linear stimulus intensity. The process of estimating power function exponents by using this somewhat simplified method is as follows: With any given subject, conduct tests of auditory stimulus identification at any fixed sound frequency for intensity ranges of, say, $1-10 \mathrm{~dB}, 1-30 \mathrm{~dB}, \ldots$ 1-90 dB. At least three ranges are needed, and the extremes (1-10 and 1-90) should be represented. Test stimuli should be given no more frequently than every $20 \mathrm{sec}$, since the use of shorter intervals tends to increase the error for the larger stimulus ranges (unpublished results). Identification of test stimuli should be made to the nearest decibel. For each matrix, estimate $\sigma_{\text {eff }}^{2}$, the arithmetic mean row variance, without worrying about anchor effects for this estimate. Estimate $\mu_{s}$ simply. For example, for the matrix assembled from stimuli in the range $1-30 \mathrm{~dB}$, $\mu_{s}$ is nearly $10^{1.5}$; for the range $1-50, \mu_{s}$ is nearly $10^{2.5}$, and so on. Make a full logarithmic plot of $\sigma_{\text {eff }}^{2}$ versus $\mu_{s}$. The slope of this graph provides an estimate of $n$ for this sound frequency. This process is illustrated in Figure 6.

Although we worked within the modality of hearing, the process is, in principle, replicable for any prothetic modality.

\section{REFERENCES}

BAIRD, J. C. (1970). A cognitive theory of psychophysics: II. Fechner's law and Stevens' law. Scandinavian Journal of Psychology, 11, 89-102.
BAIRD, J. C. (1984). Information theory and information processing. Information Processing \& Management, 20, 373-381.

BraidA, L. D., \& DurLACH, N. I. (1972). Intensity perception: II. Resolution in one-interval paradigms. Journal of the Acoustical Society of America, 51, 483-502.

Braida, L. D., Lim, J. S., Berliner, J. E., Durlach, N. I., Rabinowitz, W. M., \& PURKs, S. R. (1984). Intensity perception: XIII. Perceptual anchor model of context-coding. Journal of the Acoustical Society of America, 76, 722-731.

Carlton, A. G. (1969). On the bias of information estimates. Psychological Bulletin, 71, 108-109.

GARNER, W. R., \& HAKE, H. W. (1951). The amount of information in absolute judgments. Psychological Review, 58, 446-459.

Houtsma, A. J. M. (1983). Estimation of mutual information from limited experimental data. Journal of the Acoustical Society of America, 74, 1626-1629.

Luce, R. D., Green, D. M., \& Weber, D. L. (1976). Attention bands in absolute identification. Perception \& Psychophysics, 20, 49-54.

MARKs, L. E. (1974). Sensory processes: The new psychophysics. New York: Academic Press.

Miller, G. A. (1955). Note on the bias of information estimates. In H. Quastler (Ed.), Information theory in psychology: Problems and methods (pp. 95-100). Glencoe, IL: Free Press.

Mori, S., \& WARD, L. M. (1995). Pure feedback effects in absolute identification. Perception \& Psychophysics, 57, 1065-1079.

Norwich, K. H. (1977). On the information received by sensory receptors. Bulletin of Mathematical Biology, 39, 453-461.

NorWICH, K. H. (1993). Information, sensation and perception. San Diego: Academic Press.

Norwich, K. H., Wong, W., \& SAgI, E. (1998). Range as a factor determining the information of loudness judgments: Overcoming small sample bias. Canadian Journal of Experimental Psychology, 52, 6370.

SAGI, E., \& Norwich, K. H. (1999). Weighing the anchor in absolute identification: A constant variance model. In P. R. Killeen \& W. R. Uttal (Eds.), Proceedings of the Fifteenth Annual Meeting of the International Society for Psychophysics (pp. 344-349). Tempe, AZ: International Society for Psychophysics.

SAGI, E., \& Norwich, K. H. (in press). Weighing the anchor in stimulus categorization. Canadian Acoustics.

SAGI, E., Wong, W., \& Norwich, K. H. (2001). Mathematical studies of the information in the stimulus-response matrix. Journal of Mathematical Psychology, 45, 99-114.

Smith, R. L., \& Brachman, M. L. (1980). Dynamic response of single auditory-nerve fibers: Some effects of intensity and time. In G. van den Brink \& F. A. Bilsen (Eds.), Psychophysical, physiological and behavioural studies in hearing: Proceedings of the Fifth International Symposium on Hearing (p. 313). Delft: Delft University Press.

Stevens, J. C., \& Marks, L. E. (1999). Stevens's power law in vision: Exponents, intercepts, and thresholds. In P. R. Killeen \& W. R. Uttal (Eds.), Proceedings of the Fifteenth Annual Meeting of the International Society for Psychophysics (pp. 87-92). Tempe, AZ: International Society for Psychophysics.

STEvens, S. S. (1956). The direct estimation of sensory magnitudesloudness. American Journal of Psychology, 69, 1-25.

West, R. L., Ward, L. E., \& Khosla, R. (2000). Constrained scaling: The effect of learned psychophysical scales on idiosyncratic response bias. Perception \& Psychophysics, 62, 137-151.

Wong, W., \& Norwich, K. H. (1997). Simulation of human sensory performance. BioSystems, 43, 189-197.

\section{NOTE}

1. In earlier works (e.g., Norwich, 1993), this inequality was written in the form $\sigma_{s}^{2} / N \sigma_{r}^{2} \ll 1$. We have absorbed the extra sampling factor. 
APPENDIX

Removing Anchor Effects (Edge Distortion)

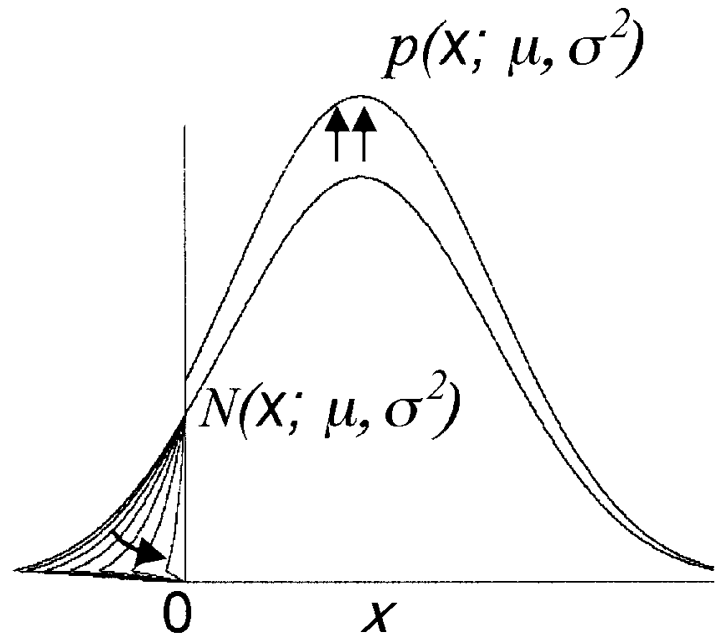

Figure A1. Schematic illustration of the process of removing the edge, or anchor, effects. A complete normal density is truncated, so to speak, by "folding over" the part of the curve that would lie to the left of $x=0$ (shown in the figure) and to the right of $x=R$ (not shown). The result is the observed normalized truncated density, containing the edge, or anchor, effect. Normalization increases the amplitude of the curve.

The full description of a process for removing anchor effects from a stimulus-response matrix is described elsewhere (Sagi \& Norwich, 1999, in press); however, we can capture the gist of the idea here. In accordance with Thurstonian theory and in agreement with our own measurements, the idealized distribution of responses along any row of a stimulus-response matrix can be represented by the normal density $N\left(x ; \mu, \sigma^{2}\right)$, where $x$ is distance along the row, $\mu$ is the row mean, and $\sigma^{2}$ is the variance common to each row. The number of rows and columns in a stimulus-response matrix corresponds to the number of stimulus and response categories, $m$, into which a given stimulus range, $R$ (in $\mathrm{dB}$ ), has been divided. For clarity, we shall represent the variables $x, \mu$, and $\sigma$ in decibels, although, in principle, they can be expressed in units of category.

At each row, the idealized distribution, $N\left(x ; \mu, \sigma^{2}\right)$, which typically extends from $-\infty$ to $+\infty$, becomes truncated or "clipped," so to speak, by the edges of the matrix. That is, $x$ can no longer vary from $(-\infty, \infty)$, but has been confined to $[0, R]$. Consequently, the probability density for each row, $p\left(x ; \mu, \sigma^{2}\right)$, becomes zero for all values outside of $[0, R]$ and requires renormalization of $N\left(x ; \mu, \sigma^{2}\right)$ for all values inside of $[0, R]$.

In other words, the area under the normal curve along any row is equal to $\int_{0}^{R} N\left(x ; \mu, \sigma^{2}\right) d x$, so that the probability density for the row can be given explicitly by the function

$$
p\left(x ; \mu, \sigma^{2}\right)=\frac{N\left(x ; \mu, \sigma^{2}\right)}{\int_{0}^{R} N\left(x ; \mu, \sigma^{2}\right) d x} .
$$

The relationship between $N\left(x ; \mu, \sigma^{2}\right)$ and $p\left(x ; \mu, \sigma^{2}\right)$ is illustrated schematically in Figure A1.

The variance of the underlying normal density, $N\left(x ; \mu, \sigma^{2}\right)$, is given by $\sigma^{2}$, and the variance of the measured density, $p(x$; $\left.\mu, \sigma^{2}\right)$, is given by $\operatorname{var}\left[p\left(x ; \mu, \sigma^{2}\right)\right]$. Clearly, these variances are similar but not identical, since $\sigma$ is constant for each row, whereas $\operatorname{var}\left[p\left(x ; \mu, \sigma^{2}\right)\right]$ differs slightly for each row. We can, however, relate one variance to the other. From the usual definition of variance we can write

$$
\operatorname{var}\left[p\left(x ; \mu, \sigma^{2}\right)\right]=\left\langle x^{2}\right\rangle-\langle x\rangle^{2}
$$

where moments about the origin are given by

$$
\left\langle x^{\lambda}\right\rangle=\int_{0}^{R} x^{\lambda} p\left(x ; \mu, \sigma^{2}\right) d x
$$

for $\lambda=1,2$. The moments on the right-hand side of Equation A2 are functions of the underlying variance, $\sigma^{2}$, so this equation can be written simply as

$$
\operatorname{var}\left[p\left(x ; \mu, \sigma^{2}\right)\right]=f\left(\mu, \sigma^{2}\right),
$$

where $f\left(\mu, \sigma^{2}\right)$ is a known function of the underlying variance. Instead of relating the underlying variance, $\sigma^{2}$, to the slightly altered variance of each row, it is more cost effective to relate $\sigma^{2}$ to a measurable value easily determined from any experimentalmatrix, the arithmetic mean row variance, $\sigma_{\text {eff }}^{2}$. In terms of $\operatorname{var}[p(x$; $\left.\mu, \sigma^{2}\right)$ ], the arithmetic mean row variance can be expressed as

$$
\sigma_{\text {eff }}^{2}=\frac{1}{R} \int_{0}^{R} \operatorname{var}\left[p\left(x ; \mu, \sigma^{2}\right)\right] d \mu .
$$

After the requisite algebra is done, the relationship between $\sigma_{\text {eff }}^{2}$ and the underlying variance $\sigma^{2}$ reduces to

$$
\sigma_{\text {eff }}^{2}=\sigma^{2}-\frac{4}{R \sqrt{2 \pi}} \sigma^{3} \text {. }
$$

Of greatest import, Equation A5 allows one to extract the underlying variance $\sigma^{2}$ from the arithmetic mean row variance $\sigma_{\text {eff }}^{2}$, obtained easily from any experimental matrix. 